\title{
Preface
}

\section{SOHO 20 - Transient events on the Sun and in the heliosphere}

This Special Issue of Annales Geophysicae contains the refereed publications of research presented at the 20th SOHO Workshop, which was held from 27 to 31 August 2007 in Ghent, Belgium, on the topic "Transient Events on the Sun and in the Heliosphere". Over 130 participants from 20 countries contributed to the success of this meeting.

Since all solar variability - from nano-flares to secular trends - is somehow "transient", defining the scientific program was quite a challenge. The sessions were organized according to the different layers of the Sun's atmosphere:

1. Transients and dynamics in the lower solar atmosphere

2. CMEs : Why and how do they erupt?

3. Heliospheric transients

4. Solar and heliospheric science in the next decade and beyond

The 4th session enabled more prospective contributions and included a panel discussion on "The next twenty years in solar physics".

It is our great pleasure to thank the members of the Scientific Organizing Committee for their help in putting together the scientific program, and the members of the Local Organizing Committee for implementing such an excellent conference, including a memorable conference dinner and a Belgian beer-tasting event.
Financial support was provided by the Belgian Science Policy, the SOHO project, ESA headquarters, and the Flemish Fund for Scientific Research. The Living With a Star program offered financial support to students.

We are grateful for the organizational and logistic support we received from the University of Ghent and from the organizing institute, the Royal Observatory of Belgium.

And finally we would like to thank all participants. It was the quality of their presentations and their contributions to the discussions that made the meeting a scientific success.

Eva Robbrecht

SIDC - Royal Observatory of Belgium

Jean-François Hochedez

SIDC - Royal Observatory of Belgium

Bernhard Fleck

ESA RSS Dept., NASA, Goddard Space Flight Center

Joseph Gurman

NASA, Goddard Space Flight Center

Robert Forsyth

Topical Editor

Published by Copernicus Publications on behalf of the European Geosciences Union. 